\title{
Flow topologies in primary atomization of liquid jets: A direct numerical simulation analysis
}

\author{
Josef Hasslberger $^{1} \dagger$, Sebastian Ketterl ${ }^{1}$, Markus Klein ${ }^{1}$, and Nilanjan \\ Chakraborty $^{2}$ \\ ${ }^{1}$ Institute of Mathematics and Applied Computing, University of the German Federal Armed \\ Forces, Werner-Heisenberg-Weg 39, 85577 Neubiberg, Germany \\ ${ }^{2}$ School of Engineering, Newcastle University, Claremont Road, Newcastle-Upon-Tyne NE1 \\ 7RU, United Kingdom
}

(Received xx; revised xx; accepted xx)

The local flow topology analysis of the primary atomization of liquid jets has been conducted using the invariants of the velocity gradient tensor. All possible small-scale flow structures are categorized into two focal and two nodal topologies for incompressible flows in both liquid and gaseous phases. The underlying Direct Numerical Simulation database was generated by the one-fluid formulation of the two-phase flow governing equations including a high-fidelity Volume-of-Fluid method for accurate interface propagation. The ratio of liquid-to-gas fluid properties corresponds to a Diesel jet exhausting into air. Variation of the inflow-based Reynolds number as well as Weber number showed that both these non-dimensional numbers play a pivotal role in determining the nature of the jet breakup, but the flow topology behavior appears to be dominated by the Reynolds number. Furthermore, the flow dynamics in the gaseous phase is generally less homogeneous than in the liquid phase because some flow regions resemble a laminarto-turbulent transition state rather than fully developed turbulence. Two theoretical models are proposed to estimate the topology volume fractions and to describe the size distribution of the flow structures, respectively. In the latter case, a simple power law seems to be a reasonable approximation of the measured topology spectrum. According to that observation, only the integral turbulent length scale would be required as an input for the a-priori prediction of the topology size spectrum.

Key words: Must be chosen during submission process

\section{Introduction}

The performance and pollutant emission of combustion devices heavily depends on the evaporation and homogeneity of the fuel-air mixture which is controlled by the preceding atomization process. It describes the disintegration of a liquid core into a large number of droplets. Further crucial technical applications can be found in medicine (e.g. asthma spray) or agriculture (e.g. manuring apparatus). The underlying physics of spray formation, especially the interaction between the turbulent flow and the liquid-gas interface, is not yet fully understood (Desjardins et al. 2013).

$\dagger$ Email address for correspondence: josef.hasslberger@unibw.de 
The development of advanced numerical techniques in the context of Direct Numerical Simulation (DNS) significantly improved our understanding of the atomization physics. Each exhibiting particular advantages and disadvantages, the Volume-of-Fluid method (Hirt \& Nichols 1981), the level-set method (Sussman et al. 1994) and the front-tracking method (Tryggvason et al. 2001) are well established for accurate interface propagation. One of the first reported Diesel spray DNS at low Reynolds and Weber number goes back to Leboissetier \& Zaleski (2001), demonstrating that no breakup occurs for laminar flow conditions inside the nozzle. Taking into account realistic turbulent conditions at the injection nozzle, DNS of a liquid sheet exhausting into gaseous atmosphere was performed by Klein (2005). For the planar water jet at the low Weber number of 270, no jet breakup and spray formation occurred. Flow and interface statistics in the near-nozzle region were also compared to experimental data revealing good qualitative agreement. Sander \& Weigand (2008) later found that not only the inflow turbulence characteristics but also the mean velocity profile at the inlet has a strong influence on the stability of liquid sheets. Accompanied by a first discussion of mesh resolution requirements in the context of two-phase DNS, Diesel jet simulations at an increased Weber number of 11,600, showing severe liquid breakup, were presented by Ménard et al. (2007). This discussion of resolution requirements for the smallest interfacial structures was continued by Desjardins \& Pitsch (2010) who studied the breakup of a temporally evolving turbulent liquid sheet at Weber numbers between 500-2,000. Herrmann (2011) investigated the primary atomization of a round Diesel jet, also highlighting the impact of finite grid resolution on interface geometry and droplet size distributions. Highly-resolved round Diesel jets at the high Weber number of 14,000, injected at laminar conditions into stagnant air, were presented by Shinjo \& Umemura (2010).

To obtain a comprehensive picture of the jet atomization phenomenon, this paper contributes by a local flow topology analysis based on the invariants of the velocity gradient tensor following the pioneering work of Chong et al. (1990); Perry \& Chong (1987). In case of incompressible flows, all possible small-scale flow structures can be categorized into two nodal and two focal topologies. To analyze the different manifestation of coherent structures, the methodology has been applied to a variety of flows including wallbounded shear flows (Chong et al. 1998) and homogeneous isotropic turbulence (Elsinga \& Marusic 2010). In the context of incompressible two-phase flows, Hasslberger et al. $(2018 b)$ analyzed the distribution of local flow topologies in bubbly air-water systems. It has been found that focal topologies, representing vortical motion, predominantly reside inside the gaseous bubbles. An example of application to a single-phase jet flow has been presented by Watanabe et al. (2014) who focused upon vortex stretching and compression near the turbulent/non-turbulent interface in a planar jet. The authors are not aware of any applications of this methodology to liquid jet atomization however. If the flow is compressible, e.g. in the context of turbulent premixed combustion (Wacks et al. 2016), the first invariant (trace) of the velocity gradient tensor assumes non-zero values. The number of possible flow topologies consequently increases from four to eight. Potential further analysis steps were demonstrated by Dopazo et al. (2007) who studied the connection between local flow topologies and local interface curvature. Including also the evolution of the invariants, an extension of the snapshot-based topology analysis has been proposed by Ooi et al. (1999). 


\section{Direct numerical simulation database}

\subsection{Numerical methodology}

The state-of-the-art two-phase solver PARIS (PArallel Robust Interface Simulator, written in Fortran, and developed by Stéphane Zaleski et al. at Institut Jean Le Rond d'Alembert, UPMC \& CNRS, Paris, France) was applied for the simulations discussed in this paper. This code is based on the one-fluid formulation of the unsteady incompressible Navier-Stokes equations including capillary forces. Two immiscible fluids are represented by a jump in density and viscosity. Propagation of the phase interface is implicitly calculated by an advection equation

$$
\frac{\partial \alpha}{\partial t}+u_{j} \frac{\partial \alpha}{\partial x_{j}}=0
$$

for the cell-based volume fraction $\alpha$ of the liquid phase. Cell-averaged fluid properties are then obtained from a weighted arithmetic mean for density and dynamic viscosity,

$$
\begin{aligned}
& \rho=\alpha \rho_{l}+(1-\alpha) \rho_{g}, \\
& \mu=\alpha \mu_{l}+(1-\alpha) \mu_{g} .
\end{aligned}
$$

The subscripts $g$ and $l$ indicate the gaseous and liquid phase, respectively. To obtain the velocity field $\boldsymbol{u}$, the momentum balance equation for two-phase flows

$$
\rho\left(\frac{\partial u_{i}}{\partial t}+\frac{\partial u_{i} u_{j}}{\partial x_{j}}\right)=-\frac{\partial p}{\partial x_{i}}+\frac{\partial \tau_{i j}}{\partial x_{j}}+\sigma \kappa n_{i} \delta_{S}
$$

is solved, in which the interface Dirac function $\delta_{S} \equiv \delta\left(\boldsymbol{x}-\boldsymbol{x}_{S}\right)$ is approximated by $|\nabla \alpha|$, and thus $\boldsymbol{n} \delta_{S}=-\nabla \alpha$, according to the Continuum Surface Force methodology proposed by Brackbill et al. (1992). The quantities $\sigma, \kappa$ and $\boldsymbol{n}=-\nabla \alpha /|\nabla \alpha|$ denote the surface tension, interface mean curvature and interface normal vector, respectively. The viscous stress tensor $\tau_{i j}$ is formulated on the basis of Stokes' hypothesis, which reduces to

$$
\tau_{i j}=\mu\left(\frac{\partial u_{i}}{\partial x_{j}}+\frac{\partial u_{j}}{\partial x_{i}}\right)
$$

for incompressible flows $(\nabla \cdot \boldsymbol{u}=0)$.

In terms of interface treatment, advanced numerical techniques are applied: a geometrical Volume-of-Fluid method including piece-wise linear interface reconstruction, and a height function method combined with continuous surface force balancing for interface curvature determination (Popinet 2018). In the framework of the finite-volume method, spatial discretization on a cubic staggered grid is implemented by the third-order QUICK scheme for momentum advection and the second-order central differencing scheme for diffusive fluxes. Volume fraction advection, Eq. 2.1, is implemented by the CIAM scheme (Scardovelli \& Zaleski 2003) (similar to the method of Parker \& Youngs (1992)) and explicit temporal discretization by a second-order accurate Runge-Kutta scheme. The projection method invokes a successive over-relaxation solver for the pressure Poisson equation. Parallelization is realized by the domain decomposition technique and MPI processor communication. Further details on the utilized numerical techniques can be found in (Tryggvason et al. 2011).

\subsection{Computational setup}

The investigated setup is similar to the configuration studied by Ketterl \& Klein (2018). For one of the examined cases, Fig. 1 visualizes the primary atomization process: The 


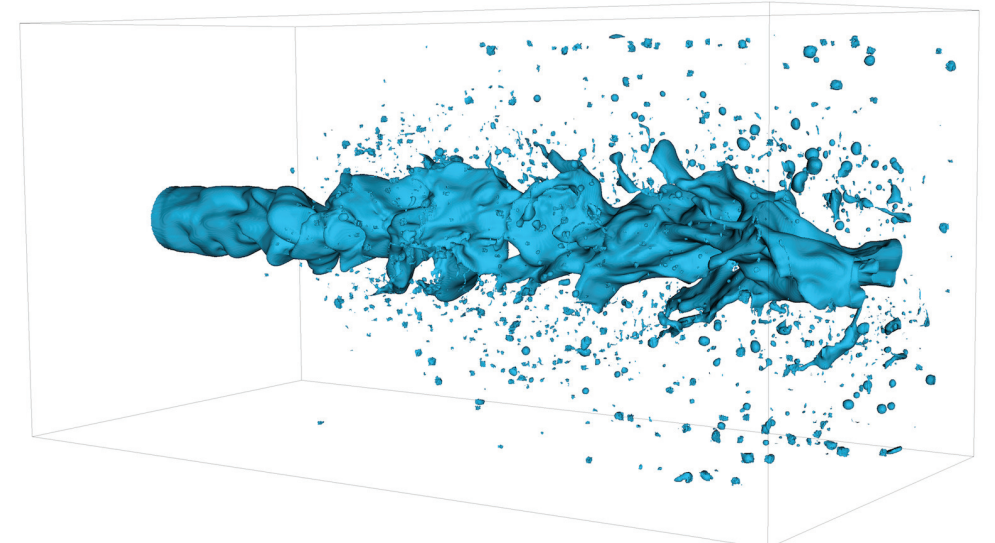

Figure 1. Three-dimensional round jet configuration: Primary atomization in the hiRe-loWe case visualized by the blue $\alpha=0.5$ iso-contour indicating the liquid-gas interface; The mean flow direction is oriented from left to right.

liquid is injected through a round nozzle on the left and the interface immediately starts to wrinkle according to the turbulent inflow conditions. Interface corrugations grow in downstream direction and ligaments are formed. These ligaments are being stretched, eventually rupture and form droplets. Secondary atomization, i.e. the breakup of droplets into smaller droplets, is naturally included in the simulation but is not at the focus of this work.

The near-field of a liquid round jet injected through a nozzle into a quiescent gas is a classical flow instability problem. Mean vorticity and its fluctuations evolve from a wall-confined stream to an unbound environment as the flow leaves the nozzle. Coherent structures (Brown \& Roshko 1974), due to the mean vorticity shedding, form, grow and interact with vorticity fluctuations downstream of the nozzle. For large enough Reynolds numbers, the flow transitions to a turbulent jet. Due to the liquid jet oscillation and due to the no-slip condition at the liquid-gas interface, pressure and viscous forces set the initially quiescent gas in motion.

The rectangular computational domain extends $12 D$ in axial direction and $6 D$ in both span-wise directions, where $D$ denotes the diameter of the round nozzle. At the side boundaries, homogeneous Neumann conditions are imposed, including a narrow band where the velocity is filtered to avoid numerical instabilities. At the outflow boundary, back-flow into the domain is prevented by clipping negative velocities to zero, also for stability reasons. Dirichlet conditions are applied at the inlet boundary. To imitate a turbulent channel flow inside the nozzle region, the mean velocity profile of Stanley et al. (2002) is superimposed by a homogeneous isotropic fluctuation level of $5 \%$ and an integral turbulent length scale of $L_{t}=D / 4$ using the digital filter method of Klein et al. (2003). This method is based on the scaling and Gaussian filtering of random fluctuations, and it reproduces first- and second-order one-point statistics as well as given length scales.

Corresponding to Diesel engine conditions at a pressure of around $5.2 \mathrm{MPa}$ and a temperature of around $900 \mathrm{~K}$, the density and viscosity ratio are specified to be $\rho_{l} / \rho_{g}=40$ and $\mu_{l} / \mu_{g}=40$, respectively. It follows that there is no jump of kinematic viscosity at the phase boundary, i.e. $\nu=\nu_{l}=\nu_{g}$. The liquid viscosity $\mu_{l}$ and the surface tension $\sigma$ are adjusted to achieve the desired values of the inflow-based Reynolds number $R e=$ $\rho_{l} u_{0} D / \mu_{l}$ and Weber number $W e=\rho_{l} u_{0}^{2} D / \sigma$ in Tab. 1 , respectively.

Spatial discretization by a uniform Cartesian mesh of grid size $\Delta x=\Delta y=\Delta z=D / 64$ 
yields an overall number of $768 \times 384 \times 384 \approx 1.13 \times 10^{8}$ computational cells. According to the universal equilibrium theory by Kolmogorov, the smallest dissipative length scale that has to be resolved for DNS can be estimated by $\eta \sim L_{t} R e_{t}^{-3 / 4}$ using the integral turbulent length scale $L_{t}$ and the turbulent Reynolds number $R e_{t}=u^{\prime} L_{t} / \nu_{l}$ (Batchelor 1953). Inserting the prescribed inflow characteristics for $u^{\prime}$ and $L_{t}$ yields $\eta / \Delta x$ values between 0.68 and 1.35 , cf. Tab. 1. Thus, the grid size is of the order of the Kolmogorov length scale in all investigated cases, and should be sufficient to resolve small-scale flow topologies. It is hardly possible to draw further conclusions from the given values of $\eta / \Delta x$ because their calculation is based on dimensional considerations rather than being exact.

Manual mesh refinement (in one coordinate direction) is not a convenient choice because the interface is moving heavily showing arbitrary alignment with the underlying mesh and including strong topological changes. Alternative strategies like adaptive mesh refinement involve new issues like flux splitting at the boundary between different refinement levels as well as load balancing in a massively parallel environment. Furthermore, the theoretical order of discretization is difficult to maintain for local mesh refinement. The usage of uniform meshes is a standard procedure for solvers, which are based on a geometrical Volume-of-Fluid method since this method provides some kind of subgrid treatment of the interface (Ling et al. 2017; Ménard et al. 2007). There are further practical aspects related to the meshing strategy. The implementation of special-purpose post-processing tools (like the topology analysis in this work) is relatively straightforward for uniform Cartesian meshes. Any kind of interpolation introduces a new source of uncertainty.

Resolution of the Kolmogorov scale might not be sufficient in the context of two-phase flow DNS simulations. Turbulent structures are believed to follow cascade dynamics such that the smallest flow structures can be determined based on dimensional arguments. However, primary atomization is a more complex problem. Especially the stretching and breakup of thin ligament structures is a highly non-linear process involving strong topological changes, and does not necessarily follow a cascade process (Ling et al. 2015). It is a matter of ongoing discussion how to determine the required mesh density in terms of accurate representation of interface dynamics. There are empirical criteria (e.g. by Desjardins \& Pitsch (2010)) to evaluate the grid spacing with respect to droplet stability resolution requirements. Nevertheless, a universal criterion, e.g. based on dimensional arguments, has not been found so far. Furthermore, the required resolution always depends on the kind of information to be extracted from the simulation. First- and second-order statistics like mean flow and fluctuation profiles converge faster than higherorder statistics. For very sensitive statistics like droplet size distributions, it may even be impossible to achieve a grid-independent solution as argued by Herrmann (2011) and Ling et al. (2017).

The same point in time is compared for all four cases. Considering the constant time step $\Delta t$, which yields a CFL number of approximately 0.3 , the analyzed snapshot after $15,000 \Delta t$ corresponds to approximately 3.5 flow-through times based on the centerline velocity.

\section{Mathematical background}

According to Perry \& Chong (1987); Chong et al. (1990), amongst others, the invariants of the velocity-gradient tensor

$$
A_{i j} \equiv \frac{\partial u_{i}}{\partial x_{j}}=S_{i j}+W_{i j}
$$




$\begin{array}{lccc}\text { Case } & R e & W e & \eta / \Delta x \\ \text { loRe-loWe } & 2,000 & 2,000 & 1.35 \\ \text { hiRe-loWe } & 5,000 & 2,000 & 0.68 \\ \text { loRe-hiWe } & 2,000 & 5,000 & 1.35 \\ \text { hiRe-hiWe } & 5,000 & 5,000 & 0.68\end{array}$

TABle 1. Case overview in terms of the inflow-based Reynolds number Re, Weber number $W e$, and estimated ratio of Kolmogorov length scale to grid spacing $\eta / \Delta x$

give rise to a set of local flow topologies. Here, $S_{i j}=0.5\left(A_{i j}+A_{j i}\right)$ and $W_{i j}=0.5\left(A_{i j}-\right.$ $\left.A_{j i}\right)$ represent the tensor's symmetric and anti-symmetric components, respectively. The corresponding characteristic equation $\lambda^{3}+P \lambda^{2}+Q \lambda+R=0$ exhibits three solutions, i.e. the eigenvalues $\lambda_{1}, \lambda_{2}$ and $\lambda_{3}$ of $A_{i j}$. Since the first invariant of $A_{i j}$ is given by

$$
P \equiv-\operatorname{trace}\left(A_{i j}\right)=-\left(\lambda_{1}+\lambda_{2}+\lambda_{3}\right)=-S_{i i}=0
$$

for incompressible flows $(\nabla \cdot \boldsymbol{u}=0)$, the second and third invariant of $A_{i j}$ reduce to

$$
Q \equiv\left(\operatorname{trace}\left(A_{i j}\right)^{2}-\operatorname{trace}\left(A_{i j}^{2}\right)\right) / 2=\left(-S_{i j} S_{i j}+W_{i j} W_{i j}\right) / 2
$$

and

$$
R \equiv-\operatorname{det}\left(A_{i j}\right)=\left(-S_{i j} S_{j k} S_{k i}-3 W_{i j} W_{j k} S_{k i}\right) / 3 .
$$

The characteristic equation's discriminant

$$
D=\left(27 R^{2}+4 Q^{3}\right) / 108
$$

divides the $Q-R$ phase-space into two regions: $D>0$ where $A_{i j}$ shows one real eigenvalue and two complex conjugate eigenvalues (and therefore focal topologies), and $D<0$ where $A_{i j}$ shows three real eigenvalues (and therefore nodal topologies). Corresponding to $D=0$, two lines separating the topologies in the phase-space are given by

$$
\begin{aligned}
& r_{1 a}=-2(-3 Q)^{3 / 2} / 27, \\
& r_{1 b}=+2(-3 Q)^{3 / 2} / 27 .
\end{aligned}
$$

In the region $D>0, A_{i j}$ has purely imaginary eigenvalues on the line $r_{2}=0$. The lines $r_{1 a}, r_{1 b}$ and $r_{2}$ divide the $Q-R$ phase-space into four flow topologies in the incompressible case. Both the topology borders $r_{1 a}, r_{1 b}$ and $r_{2}$ in the $Q-R$ phase-space, as well as a graphical representation of topologies $\mathrm{S} 1$ to $\mathrm{S} 4$, are shown in Fig. 2. To simplify the interpretation, the second invariant of $A_{i j}, Q$, can be split into two parts:

$$
Q=Q_{S}+Q_{W}=-S_{i j} S_{i j} / 2+W_{i j} W_{i j} / 2
$$

where $Q_{S}$ and $Q_{W}$ denote the second invariant of $S_{i j}$ and $W_{i j}$, respectively. $Q_{S}$ is always a negative quantity since $-4 \nu Q_{S}=2 \nu S_{i j} S_{i j}$, i.e. the dissipation of kinetic energy into heat per unit mass. The latter part $Q_{W}$ is directly related to vorticity $\boldsymbol{\omega}$ and enstrophy $\Phi$ according to

$$
W_{i j} W_{i j} / 2=\omega_{i} \omega_{i} / 4=\Phi / 2 .
$$

Thus, $Q<0$ is indicative of strain-dominated regions and $Q>0$ is indicative of vorticitydominated regions. 

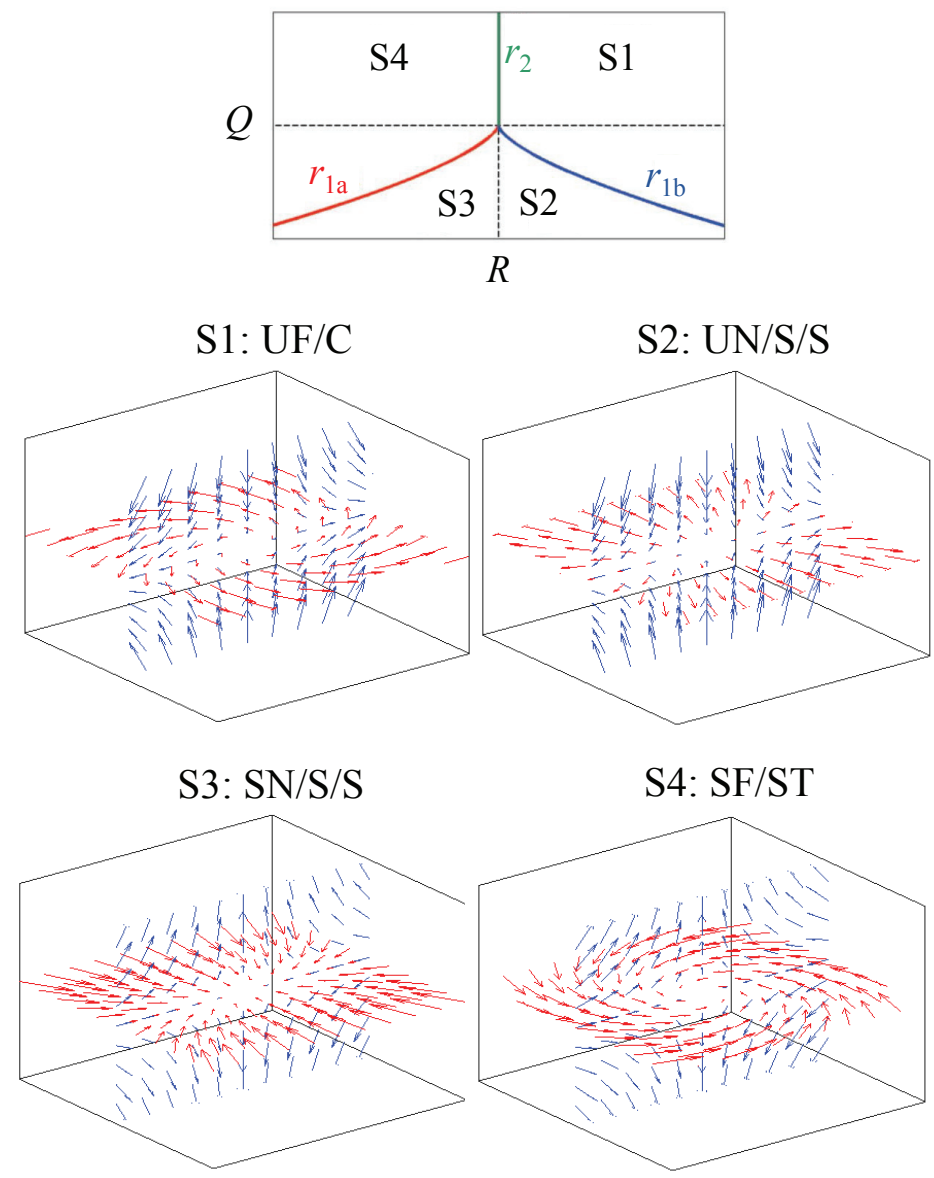

Figure 2. Classification of topologies S1 to S4: Projection of topology borders $r_{1 a}, r_{1 b}$ and $r_{2}$ in the $Q-R$ plane (top). Dashed lines indicate $Q=0$ and $R=0$, respectively; Graphical representation (bottom) corresponding to $\mathrm{UF}=$ unstable focus, $\mathrm{UN}=$ unstable node, $\mathrm{SN}=$ stable node, $\mathrm{SF}=$ stable focus, $\mathrm{C}=$ compressing, $\mathrm{S}=$ saddle, $\mathrm{ST}=$ stretching.

\section{Results and discussion}

\subsection{Vorticity field}

Since vortical motion is inherently connected to the nature of turbulence, a first impression of the atomization flow dynamics is given by means of the plane-normal component of the vorticity, i.e. $\omega_{z}=\partial u_{y} / \partial x-\partial u_{x} / \partial y$ in Fig. 3 and $\omega_{x}=\partial u_{z} / \partial y-\partial u_{y} / \partial z$ in Fig. 4. The slices in Fig. 4 represent two different stages of the jet breakup process. The vorticity and topology distributions are obviously strongly linked as elaborated in Sec. 3. Some aspects of the flow are similar in all four cases:

Counter-rotating vortex pairs can wrinkle and pinch off liquid structures. However, separated liquid structures can induce vortex pairs in a similar manner. Both phenomena can be identified in Fig. 3. The causality is not unambiguous in this context. Besides, it is difficult to draw consistent conclusions from two-dimensional slices since the considered structures, e.g. thin ligaments, do not necessarily align with the observation plane. In general, the liquid structures exhibit a strongly three-dimensional shape. Lasheras \& Choi (1988) experimentally showed that stream-wise and span-wise vortices are interacting and 
leading to a three-dimensional turbulent planar mixing layer. In the round jet configuration investigated here, azimuthal (i.e. vortex rings) and axial vorticity components are interacting as is evident from Fig. 5. Since the behavior is qualitatively in agreement with the other cases, only the loRe-hiWe case is shown. In order to accentuate dominant focal structures (referring to topology $\mathrm{S} 1$ or $\mathrm{S} 4$ ), the three-dimensional $Q=5$ iso-contour is displayed. In the high-Reynolds number cases, the focal structures are even smaller and harder to distinguish.

Moreover, noticeable vorticity production occurs in the shear layers close to the inlet, where the jet is still compact. It is well known that vorticity is produced in shear layers (Brown \& Roshko 1974). This is exactly the situation in the vicinity of the nozzle where a strong vertical gradient of axial velocity, $\partial u_{x} / \partial y$, can be found. In this region, the gas is initially at rest whereas the liquid velocity is still close to the inlet velocity. As can be seen, the sign of the induced vorticity is reversed on opposite sides of the jet. This observation might be interpreted as vortices wrapping around the liquid jet.

The vorticity level in the liquid phase is clearly lower than in the gaseous phase, which is characterized by a lower density but also lower viscosity. Tripathi et al. (2014) claim that vorticity accumulates in the lighter fluid independent of the viscosity ratio and the concaveness/convexness of the interface which follows from stability arguments (Dixit \& Govindarajan 2010). In order to exclude the viscosity ratio being responsible for this behavior, Tripathi et al. (2014) imposed identical viscosities in their simulation and observed the same behavior. Dopazo et al. (2000) present an analytically exact instantaneous relation between the vorticity values immediately above and below the interface, as a function of the strain-rate tensor components and the viscosity ratio. However, using a linearized prediction equally applicable to the vorticity values immediately above and below the interface, Farsoiya et al. (2017) found that the vorticity jump across the interface generally depends on both the density and viscosity ratio of the two fluids. Both statements from literature do not contradict an additional vorticity accumulation effect due to density differences, in unsteady turbulent flows and in regions sufficiently away from the interface.

In addition, the jet half width (radial distance at which the considered variable drops to $50 \%$ of the corresponding mean centerline value) with respect to the velocity field is clearly larger than with respect to the liquid volume fraction.

As a first approximation, the effects of the variation of Reynolds and Weber number (cf. Tab. 1) on jet breakup seem to be comparable in the sense of the intact liquid core length and the number of separated ligaments or droplets. Strongest breakup consequently appears in the high-Reynolds high-Weber case. Increasing the Weber number means that the effect of surface tension forces is reduced compared to inertia effects. Accordingly, interfacial structures are less rounded off and tend to develop thin ligaments with sharp edges. That behavior can also be observed in Fig. 3. In contrast, the size of turbulent flow structures in the gaseous phase is rather determined by the Reynolds number. According to the common understanding, smaller structures are expected at higher values of the Reynolds number. This behavior is not directly evident since the enhanced jet breakup might induce additional turbulent motion in the gaseous phase.

\subsection{Topology field}

Figure 6 shows the result of the invariants-based topology analysis introduced in the mathematical background section. The topology field, consisting of focal topologies S1 (blue) and S4 (purple) as well as nodal topologies S2 (red) and S3 (orange), confirms the impression from the vorticity field that the size of flow structures is mainly determined by the Reynolds number. Agreeing with the cascade process in turbulent flows, a spectrum 

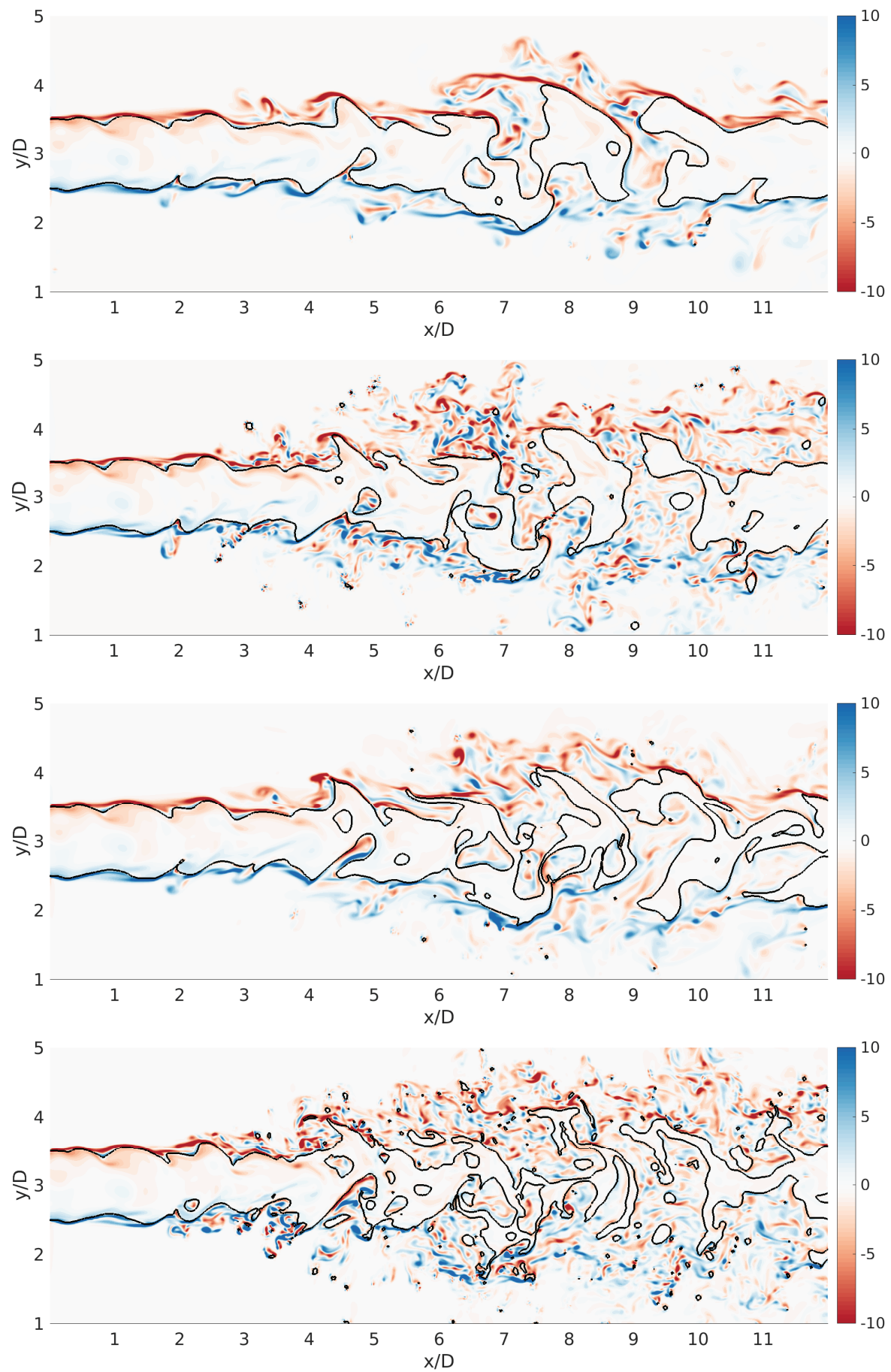

FiguRE 3. Slices of the plane-normal component of vorticity with the black line indicating the phase interface; From top to bottom: loRe-loWe, hiRe-loWe, loRe-hiWe, hiRe-hiWe. 

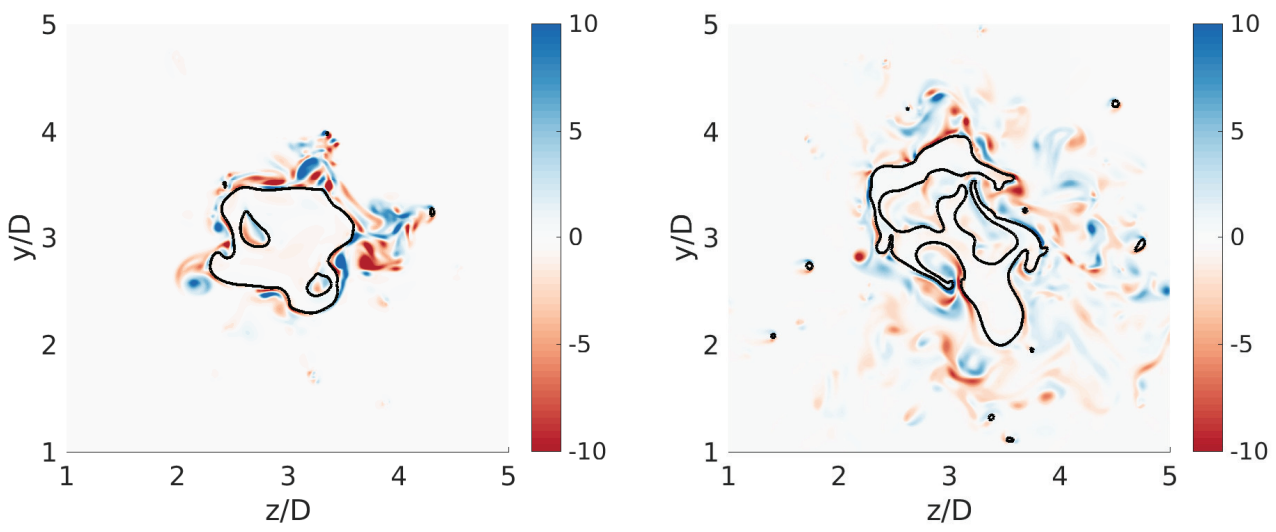

FIGURE 4. loRe-hiWe case: Slices of the plane-normal component of vorticity with the black line indicating the phase interface, for two different axial locations: $x / D=4$ (left) and $x / D=8$ (right).

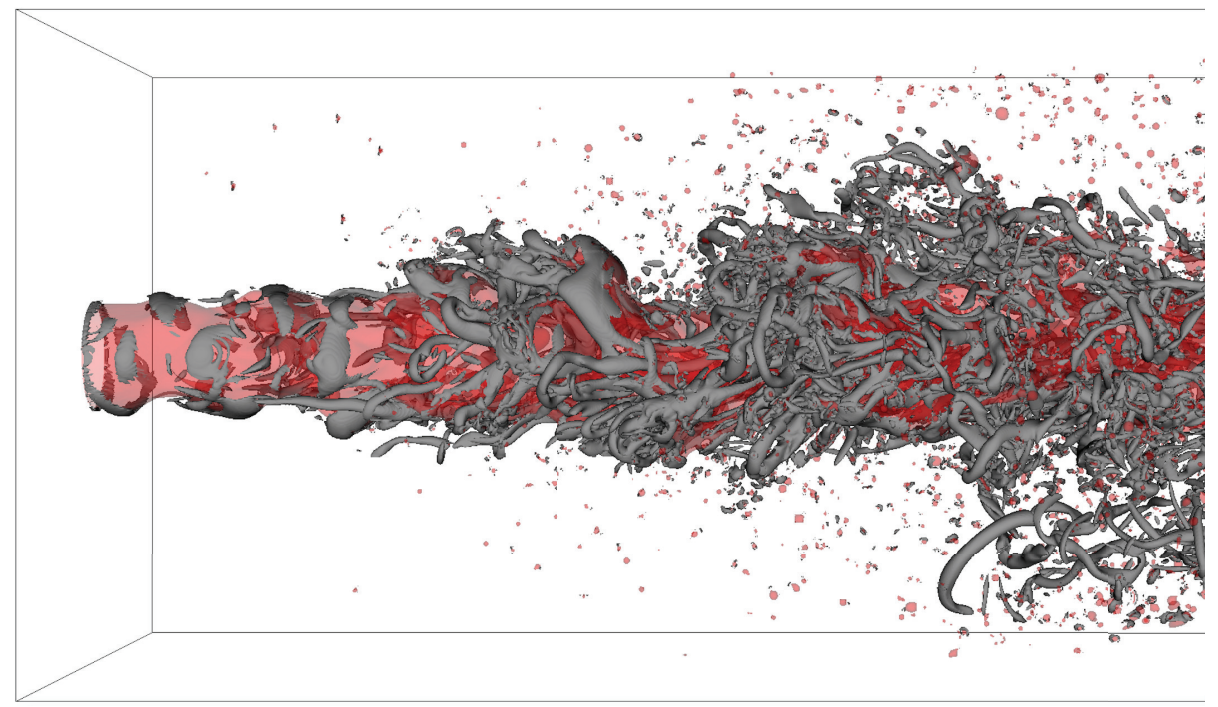

Figure 5. loRe-hiWe case: Three-dimensional iso-contours of $\alpha=0.5$ (semi-transparent red) indicating the phase interface and $Q=5$ (grey) indicating dominant focal structures.

of topology island sizes can be observed, which will be quantified in Sec. 4.4. It should be kept in mind that the kinematic viscosity $\nu$ and therefore the Reynolds number based on inflow conditions, $R e=u_{0} D / \nu$, is identical in both phases. However, the turbulent Reynolds number $R e_{t}=u^{\prime} L_{t} / \nu$ varies locally and appears to be higher in the gaseous phase. Examining DNS statistics of a three-dimensional two-phase jet configuration at a similar inflow-based Reynolds number (Klein 2005), it may roughly be estimated that $u_{l}^{\prime} / u_{g}^{\prime} \approx 0.2$ and $L_{t, l} / L_{t, g} \approx 0.5$. Accordingly, $R e_{t, l} / R e_{t, g} \approx 0.1$ - a difference of approximately one order of magnitude in terms of the turbulent Reynolds number.

Another interesting observation from Fig. 6 is the periodic occurrence of nodal S2 structures near the points of maximum local interface deflection on the gas side in the vicinity of the interface near the nozzle. In an independent study on the topology distribution in incompressible bubbly flows, Hasslberger et al. $(2018 b, a)$ observed a switch 
from nodal topology S2 to nodal topology S3 when the character of the flow around the bubble changes from diverging (up to the bubble center) to converging (behind the bubble center), respectively. In both the case from literature and the case investigated here, the switch of nodal topologies occurs at the position of maximum local interface deflection.

The liquid flow structures in the vicinity of the inlet nozzle are similar in all cases since the synthetic turbulent inflow velocity and the analyzed time snapshot are identical. The focal topologies at the left boundary, outside of the nozzle region, must be assessed as spurious behavior which stems from the ill-conditioned velocity gradient tensor in that region. This nearly laminar flow region is in fact a correct solution of the Navier-Stokes equations, i.e. the flow solution itself does not show any spuriousness. Since the topology classification is local, spurious identification in some regions does not affect the results in the rest of the computational domain. Provided that the velocity gradients are nonzero (satisfied everywhere except in the mentioned region), the topology classification is correct.

\subsection{Axial volume fraction distribution}

The axial development of plane-averaged (with respect to both span-wise directions) topology volume fractions is depicted in Fig. 7. The data is evaluated in a conditional manner, i.e. separately for the gaseous phase (left column) and the liquid phase (right column). In the gaseous phase, from inlet to outlet, the share of focal topologies S1 and S4 increases at the expense of nodal topologies S2 and S3 as vortical motion is continuously induced by the mechanisms mentioned in Sec. 2.2. It is worth noting that a coupling of azimuthal and axial vorticity filaments, as displayed in Fig. 5, is involved in the nodalto-focal transition process in axial direction. Whereas the general behavior is the same in all cases, the tendency towards an asymptotic state with respect to the volume fraction distribution scales both with $R e$ and $W e$. As discussed above, the size of topology islands scales primarily with $R e$ but the corresponding volume fractions seem to depend also on $W e$. Regarding the global exchange from nodal topology S3 to nodal topology S2 in axial direction, the gas entrainment from the surrounding atmosphere of the jet must be taken into account. There is a net inflow via the side boundaries of the domain which, for small values of $x / D$, gives rise to a globally converging flow situation in the gaseous phase (corresponding to S3). The entrainment increases in axial direction (note that there is a no-slip condition at the inflow plane everywhere except the nozzle) and saturates roughly where the liquid jet starts breaking up, at around $x / D=5$. The inward-directed radial flow at the lateral boundaries is countered by the breakup and spreading of the jet flow, resulting in local diverging flow situations surrounding the liquid core (corresponding to S2). Figure 6 shows that the major part of converging S3 topologies can be found at the lateral boundaries towards the nozzle side, whereas diverging S2 topologies are mainly found at the edge of the developing and expanding shear flow. Accordingly, the dominant nodal topology changes from S3 to S2 at a certain distance from the nozzle. In the investigated setup, the crossover point occurs approximately at half of the axial domain length but the location generally depends on the size of the computational domain in radial direction. Nevertheless, the physical processes are unaffected by the domain setup.

In the liquid phase, only marginal changes in axial direction can be observed. Due to the fluctuating nature of the two-phase jet flow, the topology shares are oscillating but their means remain at a fairly constant level. Note that the behavior directly at the nozzle is prescribed by the synthetic turbulent inflow data. In all cases, the volume fraction is at around $35 \%$ for focal topologies S1 and S4, somewhat below $20 \%$ for nodal topology S2, and around $10 \%$ for nodal topology S3.

The following theoretical discussion on incompressible homogeneous isotropic turbu- 

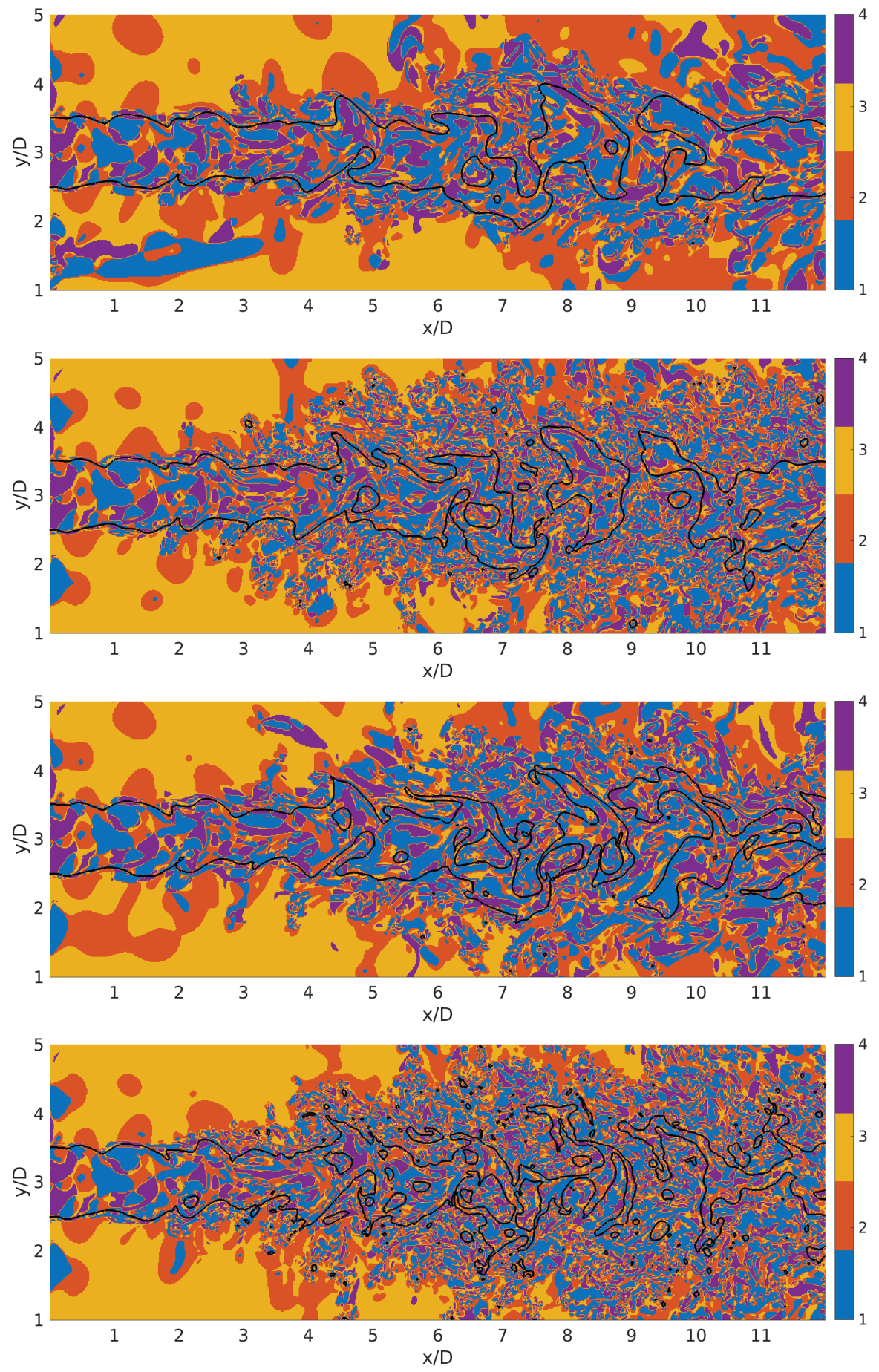

Figure 6. Slices of the flow topology field where 1-4 in the color bar refer to S1-S4 respectively; The black line indicates the phase interface; From top to bottom: loRe-loWe, hiRe-loWe, loRe-hiWe, hiRe-hiWe. 

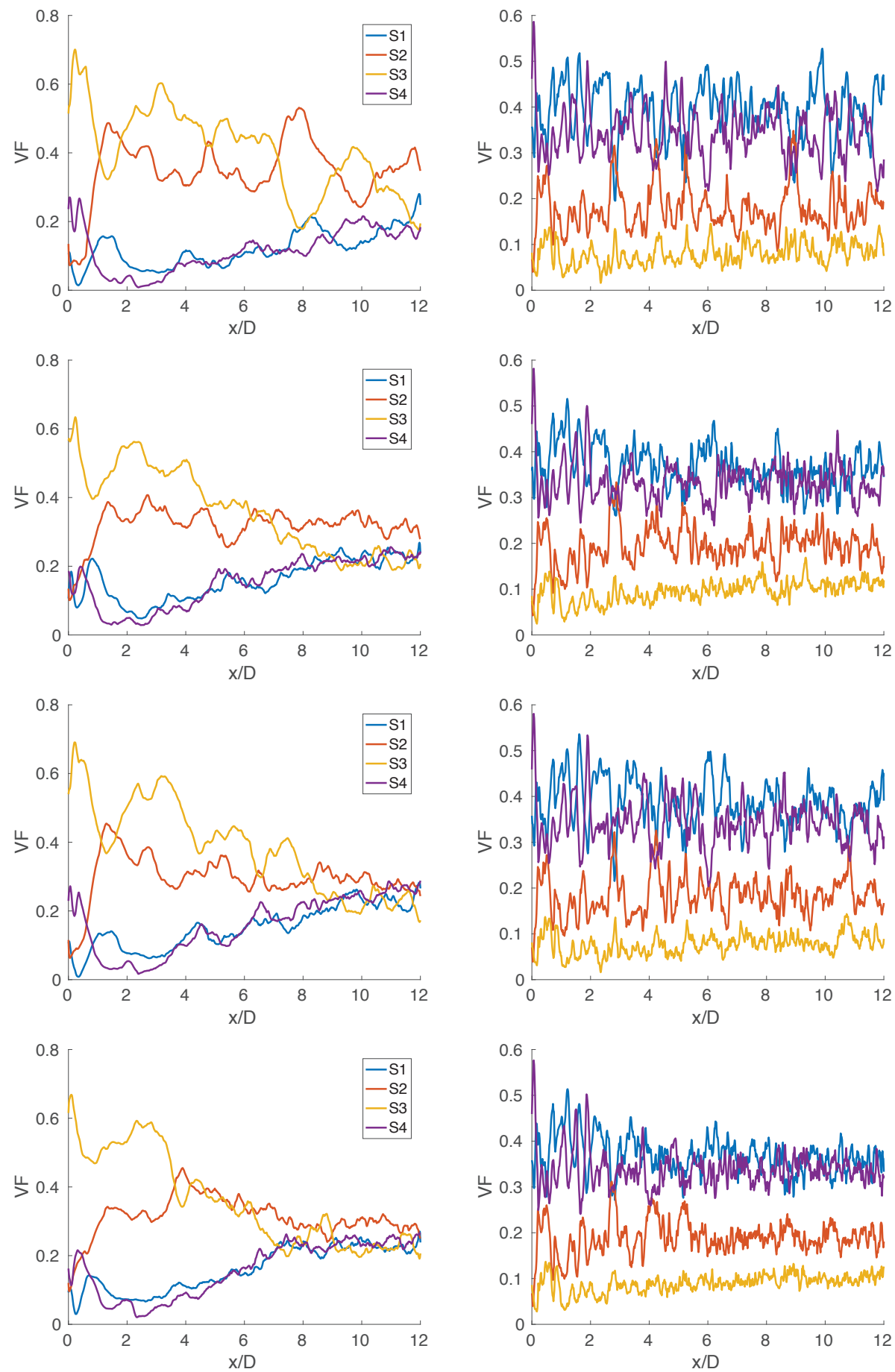

Figure 7. Axial volume fraction distribution of focal topologies S1 and S4 as well as nodal topologies S2 and S3 for the gaseous phase (left) and liquid phase (right); From top to bottom: loRe-loWe, hiRe-loWe, loRe-hiWe, hiRe-hiWe. 


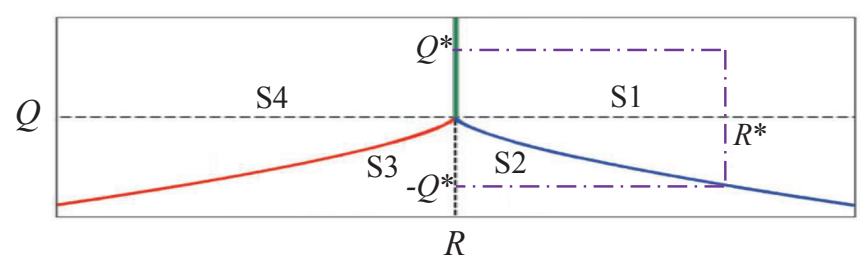

FigURE 8 . Incompressible topology map including the topology borders $r_{1 a}$ (red line), $r_{1 b}$ (blue line), $r_{2}$ (green line), and the hypothetical boundary between populated and unpopulated regions (dash-dotted purple line). Dashed black lines indicate $Q=0$ and $R=0$, respectively.

lence serves as a reference for comparison. The topology border relationship

$$
r_{1 b}(Q) \propto(-3 Q)^{3 / 2}
$$

reflects the scaling behavior of the second and third invariant, cf. Sec. 3. $Q\left(C \cdot A_{i j}\right)=$ $C^{2} \cdot Q\left(A_{i j}\right)$ and $R\left(C \cdot A_{i j}\right)=C^{3} \cdot R\left(A_{i j}\right)$ hold for any arbitrary constant $C \in \mathbb{R}^{+}$since $\operatorname{trace}\left(C \cdot A_{i j}\right)=C \cdot \operatorname{trace}\left(A_{i j}\right)$ and $\operatorname{det}\left(C \cdot A_{i j}\right)=C^{3} \cdot \operatorname{det}\left(A_{i j}\right)$. In other words, scaling of a reference flow solution to obtain different levels of velocity fluctuation $u^{\prime}$ or integral length scale $L_{t}$ does not change the classification of each sample in the topology diagram. The corner point $\left(Q^{*}, R^{*}\right)$ of a hypothetical rectangle, which is lying on the mentioned topology border (cf. Fig. 8), is given by

$$
R^{*}=r_{1 b}\left(Q=Q^{*}\right)=2 / 27\left(-3 Q^{*}\right)^{3 / 2} .
$$

Let us now assume that the rectangle represents the boundary between heavily populated (towards the origin) and poorly populated (towards infinity) regions in the topology diagram. In realistic flows, local deviations from the rectangular shape are likely to exist but may well annihilate each other in terms of the populated area. The populated area $\Omega_{\mathrm{S} 2}$ corresponding to topology $\mathrm{S} 2$ can then be calculated by

$$
\Omega_{\mathrm{S} 2}=\left|\int_{0}^{Q^{*}} r_{1 b}(Q) \mathrm{d} Q\right|=4 / 405\left(-3 Q^{*}\right)^{5 / 2} .
$$

The assumption of a homogeneous isotropic flow suggests a symmetric population in the $Q-R$ space such that the populated overall area $\Omega$ reads

$$
\Omega=\sum_{k=1}^{4} \Omega_{\mathrm{S} k}=2 Q^{*} \cdot 2 R^{*} .
$$

The presented ansatz finally allows for the calculation of the different volume fractions,

$$
\begin{gathered}
\mathrm{VF}_{\mathrm{S} 2}=\mathrm{VF}_{\mathrm{S} 3}=\Omega_{\mathrm{S} 2} / \Omega=10 \%, \\
\mathrm{VF}_{\mathrm{S} 1}=\mathrm{VF}_{\mathrm{S} 4}=\left(\Omega / 2-\Omega_{\mathrm{S} 2}\right) / \Omega=40 \%,
\end{gathered}
$$

which are independent of the particular choice of $Q^{*}$ (and therefore $R^{*}$ ). Thus, the total percentage of volume fractions in incompressible homogeneous isotropic turbulence is expected to be $20 \%$ for nodal and $80 \%$ for focal topologies. The two-phase jet configuration investigated here naturally deviates from this idealized state. Especially the nodal topology S2 is increased (at the expense of other topologies) which matches the globally diverging character of the jet flow. 


\subsection{Topology island size distribution}

For example in spray combustion, the investigation of turbulence-chemistry interaction is an outstanding field of research. In this context, it is important to know the spectrum of turbulent length scales interacting with the flame. A flood-fill algorithm is thus applied here to determine the size distribution of the topology islands. Regarding the connectivity definition of discrete topology fields, three possibilities naturally arise. Eventually, the usage of face neighbors (6 potential neighbors of each cell not residing at the domain boundary) was preferred over edge neighbors (18 potential neighbors) and point neighbors (26 potential neighbors). Especially when using point neighbors, a disproportionately strong neighborhood connectivity was observed. Owing to the exponential character of the size distribution, the size range is partitioned into 50 exponentially sized bins. At the lowest level, the island size is limited to three finite-volume cells to exclude single- and double-cell islands which are likely to be influenced by numerical effects.

Inspecting the result in Fig. 9, where $N$ is the number of topology islands of a certain size $V=V^{*} /(\Delta x)^{3}$ where $V^{*}$ is the actual topology island volume, several interesting observations can be made: Most prominently, the size distribution follows roughly a linear trend in the log-log diagram for all four cases and it appears to be consistently higher in the gaseous phase (left column) than in the liquid phase (right column). Outliers, not following the linear trend, occur scarcely at large island sizes for all cases and are mainly located in the laminar region, i.e. sufficiently away from the jet core. In the less homogeneous gaseous phase, the linear trend is slightly less pronounced and small differences between nodal and focal topologies can be identified. Furthermore, the number of nodal topologies S3 for small island sizes is slightly increased compared to the other topologies - an observation that has also been made in turbulent premixed combustion (Chakraborty et al. 2018).

The roughly linear size distribution in the log-log diagram suggests a power law formulation to describe the topology spectrum. Its derivation is based on the general ansatz

$$
N(V)=a V^{b}
$$

with the constants $a, b \in \mathbb{R}$. First of all, we require

$$
\int_{V_{\min }}^{V_{\max }} N(V) \mathrm{d} V=\mathrm{VF}_{\mathrm{S} k} \cdot V_{\text {total }}
$$

where $\mathrm{VF}_{\mathrm{S} k}$ is the volume fraction of the considered topology $\mathrm{S} k(k \in[1,2,3,4])$. It follows

$$
\frac{a}{b+1}\left(V_{\max }^{b+1}-V_{\min }^{b+1}\right)=\mathrm{VF}_{\mathrm{S} k} \cdot V_{\text {total }}
$$

for $b \neq-1$. For $b=-1, \ln (V)$ appears as the primitive. Considering the scale disparity in turbulent flows, it is reasonable to assume $\left|V_{\max }^{b+1}\right| \gg\left|V_{\min }^{b+1}\right|$ and therefore

$$
\frac{a}{b+1} V_{\max }^{b+1} \approx \mathrm{VF}_{\mathrm{S} k} \cdot V_{\mathrm{total}}
$$

Now, let us assume we have $\psi \in \mathbb{N}$ structures of size $V_{\max }$ :

$$
N\left(V=V_{\max }\right)=a V_{\max }^{b}=\psi .
$$

Inserting Eq. 4.11 into Eq. 4.10 to eliminate $a$ yields

$$
b \approx \frac{\psi V_{\max }}{\mathrm{VF}_{\mathrm{S} k} \cdot V_{\text {total }}}-1
$$

Using $\mathrm{VF}_{\mathrm{S} k} \cdot V_{\text {total }} \gg \psi V_{\max }$, we finally obtain $b \approx-1$ and hence $a \approx V_{\max } \psi$. 

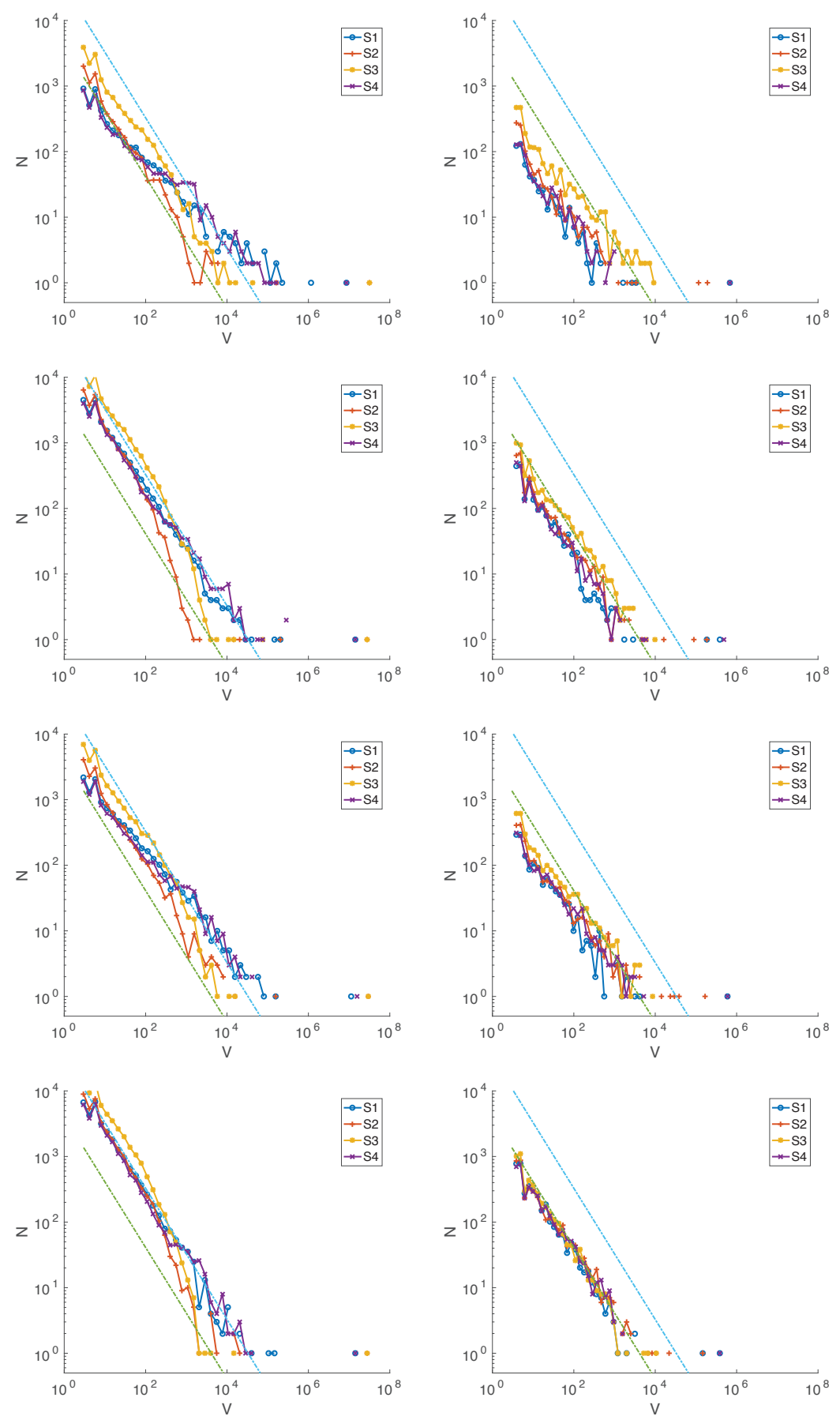

Figure 9. Topology island size distribution for the gaseous phase (left) and liquid phase (right); The turquoise and the green line represent the corresponding power law models of the gaseous phase and the liquid phase, respectively; From top to bottom: loRe-loWe, hiRe-loWe, loRe-hiWe, hiRe-hiWe. 
Alternatively, one may interpret $V$ as the actual topology island volume and the power law $N(V)=a V^{b}$ as the corresponding number density. It is then clear from dimensional arguments that $b=-1$.

To obtain a useful simple model of the spectrum, we require

$$
N\left(V=V_{\max }\right)=1
$$

which is equivalent to setting $\psi=1$. The topology size distribution then reads

$$
N(V)=V_{\max } V^{-1}
$$

and $V_{\max }=\left(L_{t} / \Delta x\right)^{3}$ is taken to be the corresponding volume measure of the integral turbulent length scale $L_{t}$. As can be seen in Fig. 9, there may exist a few larger topology islands (usually at occurrence one) but the number $N$ does not considerably increase above the volume corresponding to $L_{t}$. We know $L_{t, l} \approx D / 4$ from the prescribed inflow data and we reuse $L_{t, l} / L_{t, g} \approx 50 \%$ from Sec. 4.2 to obtain $L_{t, g} \approx D / 2$. Both models for the gaseous phase (turquoise line) and liquid phase (green line) are included in Fig. 9. Since $\mathrm{VF}_{\mathrm{S} k}$ does not enter the simple model, it does not distinguish between different topologies. Consequently, the model is identical for all four cases, only distinguishing for the gaseous and liquid phase. In a general situation, $L_{t}$ may be estimated by the nozzle diameter $D$ as a first-order approximation.

It has been seen in Sec. 4.2 that the topology field is determined by the Reynolds number rather than the Weber number. Since the total turbulent volume $V_{\text {total }}$ (i.e. the region where both focal and nodal topologies appear; somewhat larger than the spray volume) is smaller in the low-Reynolds cases, $b>-1$ must be expected in these cases according to Eq. 4.12. From a close examination of Fig. 9, the slightly different slope in the log-log diagram can be discerned because of the aforementioned effect.

The convincing performance of this simple model raises the question whether this method can be used to estimate the integral length scale $L_{t}$ when this quantity is a-priori unknown in complex flows. There is some algorithmic complexity involved to determine the topology size distribution but its advantage lies in the fact that it is based on a single time snapshot (which is usually available in contrast to long time series). Following the standard procedure, $L_{t}$ is obtained by the integration of the normalized auto-correlation tensor which requires a certain, sometimes considerable, length of the time series to guarantee a reasonable result. Using the well-established approximation $L_{t} \approx k^{3 / 2} / \epsilon$, where $\epsilon$ is the dissipation rate of the specific turbulent kinetic energy $k$, would require statistics as well.

\section{Concluding remarks}

Using a Direct Numerical Simulation database of primary jet atomization, a local flow topology analysis has been performed based on the invariants of the velocity gradient tensor. It categorizes all possible small-scale flow structures into two focal and two nodal topologies for incompressible flows.

Although both the inflow-based Reynolds number as well as Weber number are heavily influencing the jet breakup, the flow topology behavior seems to be rather dominated by the Reynolds number. As may be expected, the flow dynamics in the gaseous phase is generally less homogeneous than in the liquid phase because some flow regions resemble a laminar-to-turbulent transition state rather than fully developed turbulence. Regarding the axial development of topology volume fractions, a strong shift from nodal to focal topologies was observed in the gaseous phase. In the liquid phase, the topology shares 
remain fairly constant in axial direction (fluctuations due to the oscillating jet behavior excluded).

The discussion of DNS observations is complemented by a theoretical derivation of topology volume fractions in incompressible homogeneous isotropic turbulence. This reference solution allows for the comparison with atomization cases which only slightly deviate from this idealized state in the liquid phase. Additionally, a simple model of the size distribution of flow structures is proposed. Motivated by the roughly linear behavior of the topology spectrum in the log-log diagram, a power law ansatz is adopted. The only other input to the model is the turbulent integral length scale which is assumed to be different in both phases. Applying reverse logic, it may be possible to utilize the snapshot-based topology analysis to estimate the integral turbulent length scale when this quantity is a-priori unknown in complex flows. The robustness of this hypothesis is however yet to be assessed for a broader range of flow conditions.

Future work deals with the development of sub-grid models for the computationally more efficient Large Eddy Simulation. Observations from the topology analysis, e.g. the ratio of focal to nodal topologies, might be helpful to derive appropriate assumptions in the context of functional or structural modeling of interface-dominated two-phase flows.

\section{Acknowledgments}

Support by the German Research Foundation (Deutsche Forschungsgemeinschaft DFG, GS: KL1456/1-1 and KL1456/4-1) is gratefully acknowledged. Computer resources for this project have been provided by the Gauss Center for Supercomputing/Leibniz Supercomputing Center under grant no. pr48no. The authors also express their gratitude to the developers of PARIS for providing the source code.

\section{REFERENCES}

Batchelor, GK 1953 The theory of homogeneous turbulence. Cambridge University Press.

Brackilll, JU, Kотнe, DB \& Zemach, C 1992 A continuum method for modeling surface tension. Journal of Computational Physics 100 (2), 335-354.

Brown, GL \& Roshko, A 1974 On density effects and large structure in turbulent mixing layers. Journal of Fluid Mechanics 64 (4), 775-816.

Chakraborty, N, Wacks, D, Ketterl, S, Klein, M \& Im, H 2018 Scalar dissipation rate transport conditional on flow topologies in different regimes of premixed turbulent combustion. In 37th International Symposium on Combustion.

Chong, MS, Perry, AE \& Cantwell, BJ 1990 A general classification of three-dimensional flow fields. Physics of Fluids A: Fluid Dynamics 2 (5), 765-777.

Chong, MS, Soria, J, Perry, AE, Chacin, J, Cantwell, BJ \& Na, Y 1998 Turbulence structures of wall-bounded shear flows found using DNS data. Journal of Fluid Mechanics 357, 225-247.

Desjardins, O, McCaslin, J, Owkes, M \& Brady, P 2013 Direct numerical and largeeddy simulation of primary atomization in complex geometries. Atomization and Sprays 23 (11).

Desjardins, O \& Pitsch, H 2010 Detailed numerical investigation of turbulent atomization of liquid jets. Atomization and Sprays 20 (4), 311-336.

Dixit, HN \& Govindarajan, R 2010 Vortex-induced instabilities and accelerated collapse due to inertial effects of density stratification. Journal of Fluid Mechanics 646, 415-439.

Dopazo, C, Lozano, A \& Barreras, F 2000 Vorticity constraints on a fluid/fluid interface. Physics of Fluids 12 (8), 1928-1931.

Dopazo, C, Martín, J \& Hierro, J 2007 Local geometry of isoscalar surfaces. Physical Review E 76 (5), 056316. 
ELSINGA, GE \& MARUSIC, I 2010 Universal aspects of small-scale motions in turbulence. Journal of Fluid Mechanics 662, 514-539.

FARSOIYA, PK, MAYYA, YS \& DASGUPTA, R 2017 Axisymmetric viscous interfacial oscillationstheory and simulations. Journal of Fluid Mechanics 826, 797-818.

Hasslberger, J, Klein, M \& Chakraborty, N 2018a Flow topologies in bubble-induced turbulence: A direct numerical simulation analysis. Journal of Fluid Mechanics .

Hasslberger, J, Klein, M \& Chakraborty, N 2018b Local flow topology analysis applied to bubble-induced turbulence. In 12th International ERCOFTAC Symposium on Engineering Turbulence Modelling and Measurements. Montpellier, France.

HERRMANn, M 2011 On simulating primary atomization using the refined level set grid method. Atomization and Sprays 21 (4).

Hirt, CW \& Nichols, BD 1981 Volume of fluid (VOF) method for the dynamics of free boundaries. Journal of Computational Physics 39 (1), 201-225.

Ketterl, S \& Klein, M 2018 A-priori assessment of subgrid scale models for large-eddy simulation of multiphase primary breakup. Computers and Fluids 165, 64-77.

KLEIN, M 2005 Direct numerical simulation of a spatially developing water sheet at moderate Reynolds number. International Journal of Heat and Fluid Flow 26 (5), 722-731.

Klein, M, SAdiki, A \& Janicka, J 2003 A digital filter based generation of inflow data for spatially developing direct numerical or large eddy simulations. Journal of Computational Physics 186 (2), 652-665.

LASheras, JC \& ChOI, H 1988 Three-dimensional instability of a plane free shear layer: an experimental study of the formation and evolution of streamwise vortices. Journal of Fluid Mechanics 189, 53-86.

Leboissetier, A \& ZaLeski, S 2001 Direct numerical simulation of the atomization of liquid jet. In Proceedings of the ILASS-Europe, pp. 2-6.

Ling, Y, Fuster, D, Zaleski, S \& Tryggvason, G 2017 Spray formation in a quasiplanar gas-liquid mixing layer at moderate density ratios: a numerical closeup. Physical Review Fluids 2 (1), 014005.

Ling, Y, ZALeski, S \& ScARdovelli, R 2015 Multiscale simulation of atomization with small droplets represented by a Lagrangian point-particle model. International Journal of Multiphase Flow 76, 122-143.

Ménard, T, TAnguy, S \& Berlemont, A 2007 Coupling level set/VOF/ghost fluid methods: Validation and application to 3D simulation of the primary break-up of a liquid jet. International Journal of Multiphase Flow 33 (5), 510-524.

Ooi, A, Martin, J, Soria, J \& Chong, MS 1999 A study of the evolution and characteristics of the invariants of the velocity-gradient tensor in isotropic turbulence. Journal of Fluid Mechanics 381, 141-174.

PARKer, BJ \& Youngs, DL 1992 Two and three dimensional Eulerian simulation of fluid flow with material interfaces. Atomic Weapons Establishment.

Perry, AE \& Chong, MS 1987 A description of eddying motions and flow patterns using critical-point concepts. Annual Review of Fluid Mechanics 19 (1), 125-155.

Popinet, S 2018 Numerical models of surface tension. Annual Review of Fluid Mechanics 50, 49-75.

SANDER, W \& Weigand, B 2008 Direct numerical simulation and analysis of instability enhancing parameters in liquid sheets at moderate Reynolds numbers. Physics of Fluids 20 (5), 053301.

SCARDOVELli, R \& ZALESKI, S 2003 Interface reconstruction with least-square fit and split Eulerian-Lagrangian advection. International Journal for Numerical Methods in Fluids 41 (3), 251-274.

Shinjo, J \& Umemura, A 2010 Simulation of liquid jet primary breakup: Dynamics of ligament and droplet formation. International Journal of Multiphase Flow 36 (7), 513-532.

Stanley, SA, Sarkar, S \& Mellado, JP 2002 A study of the flow-field evolution and mixing in a planar turbulent jet using direct numerical simulation. Journal of Fluid Mechanics 450, 377-407.

Sussman, M, Smereka, P \& Osher, S 1994 A level set approach for computing solutions to incompressible two-phase flow. Journal of Computational Physics 114 (1), 146-159. 
Tripathi, MK, Sahu, KC \& Govindarajan, R 2014 Why a falling drop does not in general behave like a rising bubble. Nature Scientific Reports 4.

Tryggvason, G, Bunner, B, Esmaeeli, A, Juric, D, Al-Rawahi, N, Tauber, W, Han, J, NAS, S \& JAN, YJ 2001 A front-tracking method for the computations of multiphase flow. Journal of Computational Physics 169 (2), 708-759.

Tryggvason, G, Scardovelli, R \& Zaleski, S 2011 Direct numerical simulations of gasliquid multiphase flows. Cambridge University Press.

Wacks, DH, Chakraborty, N, Klein, M, Arias, PG \& Im, HG 2016 Flow topologies in different regimes of premixed turbulent combustion: a direct numerical simulation analysis. Physical Review Fluids 1 (8), 083401.

Watanabe, T, Sakai, Y, Nagata, K, Ito, Y \& Hayase, T 2014 Vortex stretching and compression near the turbulent/non-turbulent interface in a planar jet. Journal of Fluid Mechanics 758, 754-785. 